des Chlorsilbers in Salmiakgeist und Filtriren zurückgeblieben, wie Blei, Wismuth, Antimon, Zinn, auch Eisen (aus der angewendeten rohen Salzsäure). Die nicht zu berücksichtigenden Kupferreste waren in die ammoniakalische Lüsung übergegangen, ebenso würde etwaiges Zink in dieser Lösung verbleiben. Würden Arsen u. a. m. in der ursprünglichen Lösung vermuthet, so müsste das Chlorsilber erst ausgewaschen werden.

\title{
Ueber die Prüfung des käuflichen schwefelsauren Chinins auf fremde Chinaalkaloïde. II.
}

\author{
Von Dr. G. Kerner.
}

Im Märzhefte dieses Archivs (1880) habe ich die Fragen besprochen, welche bei der Prüfung des käuflichen schwefelsauren Chinins in Betracht kommen und im Aprilhefte folgte eine Abhandlung ,zur Geschichte des Chinidins und Cinchonidins", um die Verwirrung zu heben, welche bezüglich der Nomenclatur dieser Alkaloïde ausschliesslich nur noch in Deutschland herrschte. Auf diese beiden Veröffentlichungen hat $O$. Hesse in den Berichten der deutschen chem. Gesellschaft (20. September 1880) und im Octoberheft dieses Archivs erwidert und zwar in einer Weise, die mich wohl veranlassen könnte, seinen Auslassungen Punkt für Punkt zu folgen und an denselben, als einem Gemenge von Schmähungen, Entstellungen und Inriehtigkeiten aller Art zu zeigen, welche rühmliche Mittel Hesse versucht, nm Geschichte zu machen und anerkannte Thatsachen und Nomenclaturgebräuche, durch beliebige Zusammenwïrflung und Heranziehung irrelevanter Citate und zurechtgestutzter Nittheilungen, unklar und unentschieden erscheinen zu lassen. Wenn ich mir trotzdem den Zwang auferlege, hierauf $\%$ verzichten, so geschieht dies lediglich, um nicht in eine rein persönliche Polemik einzutreten, aus der, bei

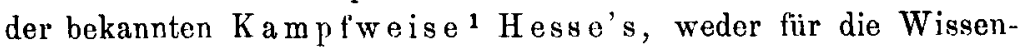

1) Zur Charakteristik derselben nur folgende kleine Probe, wie man solche auch leicht aus Hesse's Angriften auf Audere in Menge auslesen kann.

Als Beispiel dafür, dass es nach $\mathrm{Ihm}$, eine A nmassung meinerseits wäre, mich unter die Chinologen zu zäblen, "weil ich dic betreffenden 
schaft, noch für die bezüglichen praktischen Fragen irgend welche Resultate hervorgehen können. Auch für die Leser dieses Archivs wird es sich wesentlich nur um Resultate handeln, so dass ich von einer gebührenden Beleuchtung der dichterischen

Alkaloïde nicht kenne“ (man lache nicht!) führt er an, dass er ein Chininsulfat zu untersuchen Gelegenheit gehabt habe, in dem ich Cinchonin nachgewiesen, während dieses vermeintli ohe Cinchonin ,Conchinin" gewesen sei.

Mit dem gedaohten Chiningulfat verhielt es sioh aber nun, wie folgt: Ein ausserdeutsches Fabrikat (1 Büchse à 1 Kilo) kam zu meiner Beurtheilnng. Schon beim Oeffinen der Büchse war sofort zu erkennen, dass es ein mechanis ohes Gemisch darstellte, bestehend aus grosiflockigem, vollkommen probehaltigem Handelschinin, einem kleinflockigeren Sulfat und einer kleinen Menge eines specifisch schweren (beim Rütteln mebr in den unteren Theil der Büchse gelangenden) Krystallmehls. Es war daher nicht möglich auch nur ein einziges übereinstimmendes Resultat bei der quantitativen Analyse verschiedener beliebig gezogener Probeu zu erbalten. Aus dem, unter Zuhülfenahme von Loupe und Pinsel, auslesbaren pulverig-krystallinischen Theile war mit Leichtigkeit (nach Ausfälnng des Chinins durch Seignettesalz und des Chinidins durch $\mathrm{NaJ}$ ) Cinehoninsulfat bei langsamer Krystallisation in grossen Krystallen darzuatellen und untrüglich zu constatiren; in der Hauptmenge der $g$ emischten (dem kleinerflockigen Theil) war dies bei jedem Versuche nicht oder nicht so leicht (wie leicht erklärlich) möglich, wohl aber konnte darin stets deutlich $\mathrm{Chinidin} \mathrm{nachgewiesen} \mathrm{und} \mathrm{als} \mathrm{Hydrojodat} \mathrm{be-}$ stimmt werden. Duplicatanalysen der versehiedenen Theile der Waare wurden auch von meinem Assistenten, Herrn A. Kissel, mit ganz gleichem Ergebnis s ausgeführt.

Diese Wahrnehmungen theilte ich kurz (unter Einsendung einer schon beim Oeffnen der Büchse aus der Mitte derselben genommenen Probe einem Herrn mit, (seinen Namen ziehe ich absichtlich nicht in den unerquicklichen Streit) von dem Hesse sio erhalten und auch alle Umstände, die dabei in Betracht kamen, erfahren hat. Ich sehrieb ganz ausdrücklich, dass ich in dem gemischten Producte Chinidin ("Conchininsulfat") und Cinchonin fand.

Man vergleiche nun Hes $8 \mathrm{e}$ 's Darstellung und seine Verwerthung derselben mit diesem wirklichen Sachverhalt! - Er hat recht gut gewusat, dass es sich um ein gemischtes, ungleichmässiges Präparat handelte, verschweigt aber aboichtlich, dass ich a ch den Chinidingehalt desselben und zwar in ers ter Linie genannt habe. Nur auf den Umstand, dass er in dem kleineren Muster nicht a u ch das Cinchonin gefunden hat (was wohl möglich war), basirt er seine Schmähung, - und zwar unter wohlüberlegter Verschweigung und Verkehrung der Wahrheit! - Und dieser Herr hat die Stirne, Andere (so auch Dr. de Vrij) an "Wahrheitsliebe " erinnern zu wollen und mit allerlei sonstigen Verdächtigungen zu bewerfen!

Dieses eine Beispiel mag genügen, um zu zeigen, wie man Hesse's Angriffe zu beurtheilen hat. Niemand wird von mix orwarten, dass ich mich im Detail über allen seinen weiteren Unrath dieser Art verbreite. 
Fähigkeiten Hesse's Umgang nehmen und mich in Folgendem lediglich an Wesentliches halten kann. Die in meinen vorbezeichneten Abhandlungen gegebenen Daten und Ausführungen bin ich ohnehin in der Lage nach jeder Richtung ansdrücklich aufrecht zu erhalten und erlaube mir daher auf alles dort Gesagte zurückzuverweisen.

Ueber das Capitel "Chinidin" (und die Nomenclatur der vier Hsuptalkaloïde der Chinarinden) braucht überhaupt kein Wort mehr verloren zu werden, da diese Frage, auch abgesehen von meinen bezüglichen ganz objectiven Erklärungen, durch den chinologischen Congress ${ }^{1}$ in Amsterdam, sowie durch historisches und Gewohnheitsrecht bereits entschieden und abgethan ist. Will Hesse gleichwohl auf Grund seiner fixen Idee "la quinologie c'est moi" sich auch weiter seines nachgeborenen "Conchinins" erfreuen und die Confusion über den Begriff „Chinidin“ 2 weiterspinnen, so gönne man ihm dieses Privatvergnügen; wer sich wissenschaft-

1) Es ist mehr als naiv, wenn Hesse das Protokoll des Congresses gegen die Bedeutung des Beschlusses (definitive Annahmeder Pasteur's ohon Nomenclatur) und gegen mich anführen will, Das Protocoll ist nehmlich von Professor G. Planchon und $m$ i $r$ selbst verfasst, und $z$ war, wie bei solchen Referaten üblich, in möglichst bündiger Kürze, wobei man nicht speciell die einzelnen Herren (im gegebenen Falle z. B. ausser Schaer und Dr. de Vrij auch die Herren Howard, Wedell, Planchon, Phoebus, Vogl etc.) namentlich aufzuführen pflegt, welche einfach ihr Einverständniss bekundeten.

2) Das reine $\mathrm{Cb}$ inidin (Chinidinsulfat) war Seitens der Firmen Fr. Koch (als B Chinin) und C. Z immer (als $\beta$ Chinidin, Kerner 1862) läng st im Handel, als Hesse 1865 auf die Idee kam, das Alkaloïd durch Erschaffung des Namens Conchinin ,aus diesem Wirrwar herauszuziehen."?!?. Es nimmt sich daher sehr komisch aus, wenn mir Hesse , allgemeine Unkenntniss“ über diese Frage unterstellen will und dabei in der Hitze des Gefechtes den Lapsus begeht, ein meinerseits 1862 ganz deutlich als Cinchonidin charakterisirtes Alkaloüdsulfat (aus einem französischen Product erhalten) so unterschiebt, als ob ich es als Chinidin beschrieben hätte. Die Existenz der 1862 als besondere $\alpha$ und $\gamma$ Chinidin - Modificationen bezeichnete Alkaloüde habe ich bekanntlich sclbst widerrufen. Geradezu lächerlich ist es aber, wenn Hesse als Beweis für meine, nach Ihm "grundfalschen Untersuchungen “ eine $\mathrm{H}$ a $\mathrm{ndelswa}$ re anzieht, welch" Apotheker Reichel im Jahre 1858 von der Firma C. Zimmer, also zu einer Zeit erhalten hat, in welcher allerwärts noch unter der Bezeichnung „Chinidin" und "Chinidinsulfat" sowohl Chinidin - als Cinchonidinsulfat - und zufällige Gemenge Beider verkauft wurden; das fragliche zufällige Handelspräparat war also ni e ein Gegenstand einer , Untersuchung “ oder gar Veröffentlichung meinerseits gewesen! - Von di e er Qualität sind so ziemlich all e bei den Haaren herbeigeholtcn Stützen der, Erwiderungen“ Hess e's! 
lich in dieser Beziehung an seine Seite stellt, geniesst den Rubm, mit der betreffenden Nomenclatur unter den Chemikern und Chinologen aller übrigen Nationen allein zu stehen und nicht verstanden zu werden.

Ich gehe daher zur Behandlung und Beantwortung der praktischen Frage über:

Welche Anforderungen kann und hat man an ein schwefelsaures Chinin des Handels bezüglich der Prüfung a uf fremde Chinaalkaloïde zu stellen und welche Prüfungsmethode erweist sich schärfer und untrüglicher - die von mir im Jahre 1862 empfohlene (im Märzheft dieses Archivs nochmals eingehend besprochene) officinelle Ammoniakprobe, - oder die von Hesse auf Grundlage meiner Methode unter Combination mit der alten Liebig'schen Probe zusammengestellte Prüfungsweise?

Auch hierzu verweise ich zunächst auf alle Theile meiner Abhandlung (dieses Archiv Bd. XIII p. $186-205$ ) zurück und kann einfach erklären, dass Hesse's Erwiderung auch nicht eine positive Widerlegung meiner Ansführung enthält, wohl aber Beweise versucht ${ }^{1}$, die vom Kern der Sache ablenken sollen. Es wäre müssig, sich hierüber zu verbreiten, da ich weder beabsichtige, Hesse persönlich zu überzeugen, noch ferner auf eine weitere, seinerseits so unwürdig geführte Polemik einzugehen; - die letzte Instanz in allen solchen Dingen ist und bleibt das Experiment!

Jedermann kann leicht über alle diese Fragen vollkommene Klarheit erhalten, wenn er sich die

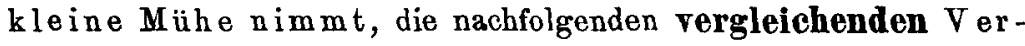
suche anzustellen.

Obschon hinlänglich bekannt, schicke ich nochmals voraus, dass sich meine Ammoniakprobe sowohl, als deren Contrefaçon von Hesse, a uf ein und dasselbe Princip stützt. Unter den neutralen Sulfaten der Chinaalkaloïde ist das schwefelsaure Chinin etwa

1) So bespricht er das Verhalten einer Lösung von 1 Theil Cinchonidinsulfat in 700 Theilen Wasser gegen Ammoniakliquor. Jedermann wird aber sofort einsehen, dass dieses Verhalten an und für sich bei der Ammoniakprobe gar nicht in Betracht kommt, da ja bei der Letzteren stets nur Lösungen grösserer oder kleinerer Antheile von Nebenakaloüdsulfaten in gesättigter ChininsulfatIösung zur Reaction gelangen. 
7 bis 10mal schwieriger in reinem Wasser von gewöhnlicher Temperatur löslich, als die übrigen Sulfate. Zieht man also ein fragliches Chininsulfat in geeigneter Weise mit der circa 10fachen Menge Wasser aus, so gelangen etwa vorhandene Sulfate der Nebenalkaloïde, wenn sie nicht mehr als einige Procente bebetragen, zuerst und zwar, wenn auch nicht absolut, so doch praktisch vollständig in Lösung, während im Uebrigen die Lösungsfähigkeit des Wassers vom reinen Chininsulfat in Anspruch genommen und erschöpft wird. Ich habe in meiner letzten Abhandlung ausführlich gezeigt und durch Analysen belegt, dass es für die Ammoniakprobe gleichgültig ist, ob man zur Extraction des Prüfungsobjectes von vornherein Wasser von gewöhnlicher Temperatur oder warmes Wasser verwendet, wenn man nurdie innige Mischung von Substanz und Waseer auf eine Normaltemperatur von praeter propter $15^{\circ} \mathrm{C}$. bringt, was zu jeder Jahreszeit sehr einfach durch Einstellen des Versuchsgefässes (unter Linsteckung eines kleinen Thermometers) in ein Becken mit frischgepumptem Brunnenwasser zu erreichen ist. Nach der Filtration haben vorkommende Luftemperaturen keinerlei Einfluss mehr anf das Resultat der Probe.

Bei der Vorschrift zur Herstellung der gesättigten Lösungen für die Ammoniakprobe habe ich das einfache Verhältniss von $1 \mathrm{Sub}$ stanz zu 10 Wasser angenommen, wie es auch in die Pharmacopöen übergegangen ist; Hesse ändert es in $1 \mathrm{Substanz} z u$ $20 \mathrm{~W}$ asser um, womit aber selbstverständlich keine Aenderung des Princips der Probe gegeben ist. Da der Wasserauszug in Bezug auf den etwaigen Gehalt an Nebenalkoïden bei letzterem Verhältniss ein verdünnterer ist, können sich höchstens nachfolgende Prüfungen damit weniger emptindlich zeigen. In Uebrigen beachte man, dass, weil also beide Proben gleichnässig in erster Linie auf der Herstellung kalt gesättigter oder vor dem Filtriren erkalteter Lösungen beruhen, in beiden Fällen überhaupt nur Das zur ferneren Reaction gelangen kann, was an fremden Alkaloïdsulfaten wirklich in diese Wasserauszüge übergegangen ist! Die Richtigkeit dieses Satzes ist festzuhalten gegenüber Hesse's neuester Behauptung, es existiren Chininsulfate mit $13 \%$ (!) Cinchonidinsulfat, in welchen er diese colossale Verunreinigung durch die Ammoniakprobe nicht habe erkennen können. Wenn dem wirklich so wäre, so ginge aus dem soeben Gesagten jedenfalls zunächst der unan- 
fechtbare Schluss hervor, dass sich dann Hesse's Probe mindestens ebenso falsch erweisen müsste; denn, wie ausserordentlich $\mathrm{scharf}$ durch die Ammoniakprobereaction Minima von Cinchonidin, Chinidin und Cinchonin in Chininsulfatlösungen und zwar auch zugleich in quantitativen Verhältnissen nachzuweisen sind, - unbestreitbar untrüglicher und sicherer als nach Hesse's Modification -, müssen folgende III Versuchsreihon überzeugend darthun.

I. Versuchsreihe. Man nehme 5-10 g. chemisch reines Chininsulfat, wie ich es als Normalchinin in meiner letzten Abhandlung beschrieben habe, ${ }^{1}$ zerreibe und übergiesse es in einem Glaskolben mit etwa 1 Liter lauwarmem destillirtem Wasser. Nach öfterem kräftigen Schütteln und mehrstündiger Digestion bringe man die Mischung durch Einstellen in kaltes Wasser auf die ungefähre Normaltemperatur von $15^{\circ} \mathrm{C}$. und filtrire nach wiederholtem Schütteln rasch durch ein trockenes Filter. Das Filtrat stellt eine kaltgesättigte wässrige Lösung von chem. reinem Chininsulfat (Normalchininlösung, abgek. NmlChL.) dar. J e 5 C.C. derselben dürfen und werden nie mehr als 3,0-3,3 C.C. Ammoniakliquor von 0,92 spec. Gew., oder $5,0-5,3$ C.C. von 0,96 spec. Grew. (officinell. AkLqr.) zum Ausfällen und genauer Wiederlösung des Chinins gebrauchen.

Mit solcher $\mathrm{NmlChL}$. bereite man nun Solutionen von genau abgewogenen kleinen Mengen Cinchonidin-, Cinchonin - und Chinidinsulfat in verschiedenen Verhältnissen und versetze je 5 C.C. (- entweder in einem in zehntel C.C. getheilten 10 C. ().-Cylinder, oder in einem Reagensglas unter Zuhülfenahme einer genauen $\mathrm{Bu}$ rette -) mit so viel Ammoniakliquor, bis die Reaction der Wie-

1) Dieses Prüparat ist nicht zu verwechseln nit einem noch so probehaltigen Handelschinin, von dem eine möglichst specifisch leichte, schön flockige Form verlangt wird. Das absolut chem. reire Chininsulfat, wie ich es hier mit Normalchinin bezeichne, hat ein ganz verschiedenes Aussehen und stellt (durch oftmaliges Umkrystallisiren aus guter Handelswaare, obne Störung der Krystallisation bereitet) cin specif. scbweres, aus durchsichtiger, spröden Nadeln bestehendes, dem Bittersalz ähnliches Salz dar.

2) Bei meinen Bestimmungen im Jahre 1862 habe ich oin möglichst reines Präparat von der Handelsform zu Grunde gelegt und jetzt das Normalchinin. Es erklären sich hieraus die Differenzen, von denen Hesse spricht. Wenn seine Nachprüfung meiner neueren Ausführungen ihn auf meine frühe ren Zablen gebracht hat, so beweist dies nur, dass or kein chem. reines Sulfat dazu benutzte. 
444 G. Kerner, Ueber d. Prüfung d. schwefols, Chinins auf fremde Chinaalkaloïde.

deriösung eintritt. Ich benutze vorzugsweise den starken Liquor von 0,92 spec. Gew., weil die Reaction damit auf den Tropfen gen a u zu treffen ist, bei dem officinellen von 0,96 spec. Gew. können Differenzen von $1 / 10$ C.C. vorkommen, was übrigens das Resultat auch nicht wesentlich stört. Aus dem Mehrverbrauch an Ammoniakliquor gegen die Normalchininlösung ergiebt sich zur Evidenz, wie sich durch die Ammoniakprobe die minimalsten Mengen von Nebenalkaloïden (meist handelt es sich nur um Cinchonidin) selbst annähernd quantitativ erkennen lassen.

Die Resultate solcher Proben werden sein, wie folgt:

(Die Temperatur aller Flüssigkeiten oder Mischungen vor der Filtration betrug $15^{\circ} \mathrm{C}$, die Lufttemperaturen schwankten zwischen 14 und $18^{\circ} \mathrm{C}$.)

Versuch 1. Normalchininlösung:
5 C.C. gebrauchen . . .

(Titrostellung des Ak-Liquors) .

Durchschnittl.

Versuch 2. 100 C. C. NrlCbL. $+0,01$ Cinchonidinsulfat. Je 5 C.C. mit je $0,0005 \mathrm{Cin}$ chonidinsulfat gebrauchen.

Durchschnittl.

Versuch 3. 100 C. C. NrlChL. $+0,025$ Cinchonidinsulfat. Je 5 C.C. mit je 0,00125 Cinchonidinsulfat gebrauchen . Durchschnittl.

Versuch 4. 100 C. C. NrlChL. $+0,05 \mathrm{Cinchonidinsulfat}$ Je 5 C.C. mit je 0,0025 Cinchonidinsulfat gebrauchen.

Durchschnittl.

Versuch 5. 100 C. C. NrlChL. $+0,10 \mathrm{Cinchonidinsulfat}$. $\mathrm{Je} 5$ C.C. mit je $0,005 \mathrm{Cin}$ chonidinsulfat gebrauchen.

Durchschnittl.

\begin{tabular}{|c|c|c|c|c|c|}
\hline \multirow{2}{*}{\multicolumn{2}{|c|}{$\begin{array}{l}\text { Verbrauch an } \\
\text { C.C.Ak bis } \\
\text { zum Eintritt } \\
\text { der Reaction } \\
\text { C.C.Ak'C.C.Ak }\end{array}$}} & \multirow{2}{*}{\multicolumn{2}{|c|}{$\begin{array}{c}\text { Differenz } \\
\text { (Mehrrer- } \\
\text { brauch) gegen } \\
\text { NrlChL. } \\
\text { C.C. } \Delta \text { k C.C. Ak }\end{array}$}} & \multirow{2}{*}{\multicolumn{2}{|c|}{$\begin{array}{l}\text { Je } 1 \text { Millig. } \\
\text { Nebenalka- } \\
\text { loüdsulfat } \\
\text { erfordern }\end{array}$}} \\
\hline & & & & & \\
\hline $\begin{array}{c}\text { von } \\
0,92\end{array}$ & $\begin{array}{l}\text { von } \\
0,96\end{array}$ & $\begin{array}{l}\text { von } \\
0,92\end{array}$ & $\begin{array}{l}\text { von } \\
0,96\end{array}$ & $\begin{array}{l}\text { ron } \\
0,92\end{array}$ & $\begin{array}{l}\text { ron } \\
0,96\end{array}$ \\
\hline 3,30 & 5,25 & & & & \\
\hline 3,30 & 5,25 & & & & \\
\hline 3,30 & 5,25 & & & & \\
\hline 3,30 & 5,25 & & & & \\
\hline$\overline{\mathbf{3}, 30}$ & $\overline{5,25}$ & & & & \\
\hline 3,45 & 5,47 & & & & \\
\hline 3,50 & 5,50 & & & & \\
\hline 3,50 & 5,45 & & & & \\
\hline 3,47 & 5,50 & & & & \\
\hline$\overline{3,48}$ & 5,48 & 0,18 & 0,23 & 0,36 & 0,46 \\
\hline 3,75 & 5,85 & & & & \\
\hline 3,75 & 5,85 & & & & \\
\hline 3,75 & 5,85 & & & & \\
\hline 3,75 & 5.85 & & & & \\
\hline 3,75 & 5,85 & 0,45 & 0,60 & 0,36 & 0,48 \\
\hline 4,10 & 6,25 & & & & \\
\hline 4,13 & 6,30 & & & & \\
\hline 4,10 & 6,30 & & & & \\
\hline 4,15 & 6,25 & & & & \\
\hline 4,12 & 6,27 & 0,82 & 1,02 & 0,32 & 0,40 \\
\hline$\{4,95$ & 7,40 & & & & \\
\hline 4,95 & 7,35 & & & & \\
\hline 5,00 & 7,40 & & & & \\
\hline 4,95 & 7,40 & & & & \\
\hline 4,98 & 7,38 & 1,68 & 2,13 & 0,33 & 0,42 \\
\hline
\end{tabular}


G. Kerner, Ueber d. Prüfung d. schwefels. Chinins auf fremde Chinaalkaloüde. 445

\begin{tabular}{|c|c|c|c|c|c|c|}
\hline & \multicolumn{2}{|c|}{$\begin{array}{l}\text { Verbrauch an } \\
\text { C.C. Ak bis } \\
\text { zum Eintritt } \\
\text { der Reaction } \\
\text { C.C.Ak|C.C. Ak }\end{array}$} & \multicolumn{2}{|c|}{$\begin{array}{c}\text { Mebrver- } \\
\text { brauch an } \\
\text { C. C. Ak gegen } \\
\text { NrlChL. } \\
\text { C.C. } \Delta \mathbf{k} \text { C.C. } \Delta \mathrm{k}\end{array}$} & \multicolumn{2}{|c|}{$\begin{array}{l}\text { Je } 1 \text { Millig. } \\
\text { Nebenalka- } \\
\text { loidsulfat } \\
\text { erfordern } \\
\text { C.C.Ak|C.C. Ak }\end{array}$} \\
\hline & \begin{tabular}{|c|} 
von \\
0,92
\end{tabular} & $\begin{array}{c}\text { von } \\
0,96 \\
\end{array}$ & $\begin{array}{c}\text { ron } \\
0,92 \\
\end{array}$ & $\begin{array}{c}\text { von } \\
0,96 \\
\end{array}$ & $\begin{array}{c}\text { von } \\
0,92 \\
\end{array}$ & $\begin{array}{c}\text { von } \\
0,96 \\
\end{array}$ \\
\hline $\begin{array}{l}\text { Versuch 6. } 100 \text { C. C. NrichL. } \\
+0,01 \text { Chinidinsulfat. } \\
\text { Je } 5 \text { C.C. mit je 0,0005 Chi- } \\
\text { nidingulfat gebrachen. }\end{array}$ & $\left\{\begin{array}{l}3,60 \\
3,60 \\
3,60 \\
3,55\end{array}\right.$ & $\begin{array}{l}5,95 \\
5,90 \\
5,90 \\
\mathbf{5 , 9 5}\end{array}$ & & & & \\
\hline Durchschuittl. & $\overline{3,58}$ & $\overline{5,92}$ & 0,28 & 0,67 & 0,56 & 1,34 \\
\hline $\begin{array}{l}\text { Versuch } 7 . \text { } 100 \text { C. C. NrlChL. } \\
+0,025 \text { Chinidinsulfat. } \\
\text { Je s C.C. mit je 0,00125 Chi- } \\
\text { nidinsulfat gebrauchen. }\end{array}$ & $\left\{\begin{array}{l}4,15 \\
4,15 \\
4,15 \\
4,15\end{array}\right.$ & $\begin{array}{l}6,70 \\
6,70 \\
6,70 \\
6,70 \\
\end{array}$ & & & & \\
\hline Durchschnittl. & 4,15 & $6, \mathbf{7 0}$ & 0,85 & 1,45 & 0,68 & 1,16 \\
\hline $\begin{array}{l}\text { Versuch 8. } 100 \text { C. C. NrlChL. } \\
\text { + 0,05 Chinidinsulfat. } \\
\text { Je } 5 \text { C. C. mit jo 0,0025 Chi - } \\
\text { nidinsulfat gebrauchen }\end{array}$ & $\left\{\begin{array}{l}5,25 \\
5,30 \\
5,30 \\
5,27\end{array}\right.$ & $\begin{array}{l}8,95 \\
9,00 \\
8,95 \\
9,00 \\
\end{array}$ & & & & \\
\hline Durchschnittl. & 5,28 & 8,97 & 1,98 & $\mathbf{3}, \mathbf{7 2}$ & 0,79 & 1,23 \\
\hline $\begin{array}{l}\text { Versuch 9. } 100 \text { C. C. NrlChL. } \\
+0,10 \text { Chinidinsulfat. } \\
\text { Je } 5 \text { C. C. mit je } 0,005 \mathrm{Chi} \text { - } \\
\text { nidinsulfat gebrauchen }\end{array}$ & $\left\{\begin{array}{l}6,95 \\
6,95 \\
7,00 \\
6,95\end{array}\right.$ & $\begin{array}{l}11,35 \\
11,30 \\
11,35 \\
11,30 \\
\end{array}$ & & & & \\
\hline Durchschnittl. & 6,96 & 11,42 & 3,66 & 6,17 & 0,79 & 1,23 \\
\hline $\begin{array}{l}\text { Versuch } 10.100 \text { C. C. NrlChL. } \\
\text { + } 0,01 \text { Cinchon insulfat. } \\
\text { Je } 5 \text { C.C. mit je 0,0005 Cin- } \\
\text { choningulfat gebrauchen }\end{array}$ & $\left\{\begin{array}{l}\mathbf{3}, \mathbf{5 0} \\
\mathbf{3 , 5 0} \\
\mathbf{3 , 5 0} \\
\mathbf{3 , 5 0}\end{array}\right.$ & $\begin{array}{l}\mathbf{5 , 6 5} \\
\mathbf{5 , 6 5} \\
\mathbf{5 , 6 5} \\
\mathbf{5 , 6 5} \\
\end{array}$ & & & & \\
\hline Durchsehnittl. & $\mathbf{3}, \mathbf{5 0}$ & 5,65 & 0,20 & 0,40 & 0,40 & 0,80 \\
\hline $\begin{array}{l}\text { Versueh 11. } 100 \text { C. C. NrlChL. } \\
\text { + } 0,025 \text { Cin choninsulfat. } \\
\text { Je } 5 \text { C. C. mit je 0,00125 Cin- } \\
\text { ch oningulfat gebranchen }\end{array}$ & $\left\{\begin{array}{l}\mathbf{3}, \mathbf{7 5} \\
\mathbf{3 , 8 0} \\
\mathbf{3 , 7 5} \\
\mathbf{3 , 7 5}\end{array}\right.$ & $\begin{array}{l}6,10 \\
6,10 \\
6,10 \\
6,10 \\
\end{array}$ & & & & \\
\hline Durchsehnittl. & $\mathbf{3 , 7 6}$ & 6,10 & 0,46 & 0,85 & $0, \mathbf{3 6}$ & 0,68 \\
\hline $\begin{array}{l}\text { Versuch } 12.100 \text { C. C. NrlChL. } \\
+0,05 \text { Cinch oninsulfat. } \\
\text { Je } 5 \text { C.C. mit je 0,0025 Cin- } \\
\text { chon insulfat gebrauchen }\end{array}$ & $\left\{\begin{array}{l}4,25 \\
4,25 \\
4,24 \\
4,30\end{array}\right.$ & $\begin{array}{l}6,85 \\
6,85 \\
6,85 \\
6,85 \\
\end{array}$ & & & & \\
\hline Durchschnittl. & 4,26 & 6,85 & 0,96 & 1,60 & 0,38 & 0,64 \\
\hline $\begin{array}{l}\text { Versuch } 13 . \\
+0,10 \mathrm{C} \text { inch oninsulfat. } \\
\text { Je } 5 \text { C. C. mit je 0,005 Cin- } \\
\text { choninsulfat gebrauchen }\end{array}$ & $\left\{\begin{array}{l}5,30 \\
5,25 \\
5,30 \\
5,25 \\
\end{array}\right.$ & $\begin{array}{l}8,40 \\
8,35 \\
8,40 \\
8,35 \\
\end{array}$ & & & & \\
\hline Durchschnittl. & 5,27 & 8,37 & 1,97 & 3,12 & 0,39 & 0,62 \\
\hline
\end{tabular}


Diese Resultate sprechen für sich selbst. Von allen drei Nebenalkaloïden wird sogar noch $1 / 2$ Millig. in 5 C.C. Chininsulfatlösung deutlich angezeigt. Cinchonin und Cinchonidin haben dabei annähernd gleiche Empfindlichkeit, während Chinidin noch weit schwerer von Ammoniak innerhalb der Chininsolution gelöst wird und die Eigenthümlichkeit zeigt, sich nach kurzem Stehen der klargewordenen Flüssigkeit wieder vollständig abzuscheiden. Es geschieht dies in Folge des Uebergangs aus der amorphen in die krystallinische, hydratwasserhaltige Form, während Cinchonidin und Cinchonin bei den Ammoniakproben in obigen Verhältnissen lange oder stets klar bleiben, wenn man die Reagensröhren verkorkt. Man prüfe nun aber noch durch eine

II. Versuchsreihe. Mischungen von chem. reinem Chininsulfat (Normalchinin) in Substanz mit bekannten kleinen Mengen Cinchonidinsulfat. Die in einem kleinen glatten Porzellanmörser gut zusammengeriebenen trockenen Mischungen übergiesse man (in dem von mir für die offic. Ammoniakprobe angegebenen Verhältniss) in kleinen verschliessbaren Kölbchen oder grossen Reagensgläsern mit 10 Gewich tsth eilen Wasser, schüttle kräftig und digerire $1 / 2-1$ Stunde. Das Wasser darf warm, lau, oder von gewöhnlicher Temperatur, ubernicht unter $15^{\circ} \mathrm{C}$. sein (vergl. Versuch 18). Vor dem Filtriren bringe man das Gemenge nur, wie mehrfach erwähnt, auf die Normaltemperatur von $15^{\circ} \mathrm{C}$.

\begin{tabular}{|c|c|c|c|c|c|c|}
\hline & \multicolumn{2}{|c|}{\begin{tabular}{|l} 
Verbrauch an \\
C. C. Ak bis \\
zum Eintritt \\
des Reaction \\
C.C. Ak.C.C.Ak
\end{tabular}} & \multicolumn{2}{|c|}{\begin{tabular}{|l} 
Mehrver- \\
brauch an \\
C.C.Akgegen \\
Nr1Chininsulf. \\
C.C.Ak|C.C.Ak
\end{tabular}} & \multicolumn{2}{|c|}{$\begin{array}{l}\text { Je } 1 \text { Millig. } \\
\text { Cinchonidin- } \\
\text { sulfat orfor- } \\
\text { dern } \\
\text { C.C.Ak|C.C.Ak }\end{array}$} \\
\hline & $\begin{array}{c}\text { von } \\
0,92\end{array}$ & $\begin{array}{l}\text { von } \\
0,96\end{array}$ & $\begin{array}{c}\text { von } \\
0,92 \\
\end{array}$ & $\begin{array}{c}\text { von } \\
0,96\end{array}$ & $\begin{array}{c}\text { von } \\
0,92 \\
\end{array}$ & $\begin{array}{l}\text { von } \\
0,96 \\
\end{array}$ \\
\hline $\begin{array}{l}\text { Versuch 14. Normalchinin- } \\
\text { sulfat }+10 \text { Wasser. } \\
\text { 5 C.C. } . \\
\text { (Titrestellung der } \Delta \mathrm{k} \text {-Liquors) }\end{array}$ & $\left\{\begin{array}{l}3,30 \\
3,35 \\
3,30 \\
3,30\end{array}\right.$ & $\begin{array}{l}5,25 \\
5,25 \\
5,30 \\
5,25 \\
\end{array}$ & & & & \\
\hline Durchschnittl. & $\mathbf{3 , 3 1}$ & 5,26 & & & & \\
\hline $\begin{array}{l}\text { Versuch 15. NrlCh-Sulfat mit } \\
\text { 1/10 } \% \text { Cinchonidinsulfat } \\
\text { verrieben. } \\
\text { Je } 5 \text { C.C. mit je } 0,0005 \text { Cin: } \\
\text { chonidinsulfat gebrauchen. }\end{array}$ & $\begin{array}{l}3,45 \\
3,45 \\
3,42 \\
3,48\end{array}$ & $\begin{array}{l}5,50 \\
5,45 \\
5,45 \\
5,45\end{array}$ & & & & \\
\hline Durchschnittl. & $\overline{3,45}$ & 5,46 & 0,14 & 0,20 & 0,28 & 0,40 \\
\hline
\end{tabular}




\begin{tabular}{|c|c|c|c|c|c|c|}
\hline & \multicolumn{2}{|c|}{$\begin{array}{l}\text { Verbrauch an } \\
\text { C.C. Ak bis } \\
\text { zum Eintritt } \\
\text { der Reaction. } \\
\text { C.C. Ak|C.C.Az }\end{array}$} & \multicolumn{2}{|c|}{$\begin{array}{l}\text { Mehrver- } \\
\text { brauch an } \\
\text { C. C. Ak gegen } \\
\text { NriChininsulf. } \\
\text { C.C.Ak C.C. Ak }\end{array}$} & \multicolumn{2}{|c|}{$\begin{array}{l}\text { Je } 1 \text { Millig. } \\
\text { Cinchonidin- } \\
\text { sulfat erfor- } \\
\text { dern } \\
\text { C.C.Ak'C.C. Al }\end{array}$} \\
\hline & $\begin{array}{l}\text { von } \\
0,92 \\
\end{array}$ & $\begin{array}{l}\text { von } \\
0,96\end{array}$ & \begin{tabular}{|l} 
von \\
0,96 \\
\end{tabular} & $\begin{array}{c}\text { von } \\
0,96 \\
\end{array}$ & $\begin{array}{c}\text { ron } \\
0,92 \\
\end{array}$ & $\begin{array}{l}\text { ron } \\
0,96 \\
\end{array}$ \\
\hline $\begin{array}{l}\text { Versuch 16. NrlCh-Sulfatmit } \\
1 / 4 \% \text { Cinchonidinsulfat } \\
\text { verrieben. } \\
\text { Je } 5 \text { C. C. mit je 0,00125 Cin- } \\
\text { chonidinsultat gebrauchen. }\end{array}$ & $\left\{\begin{array}{l}3,75 \\
3,75 \\
3,75 \\
3,75\end{array} \mid\right.$ & $\begin{array}{l}5,80 \\
5,80 \\
5,85 \\
5,85\end{array}$ & & & & \\
\hline Durchschnittl. & 3,75 & $\mathbf{5 , 8 2}$ & 0,44 & 0,56 & 0,35 & 0,44 \\
\hline $\begin{array}{l}\text { Versuch 17. NrlCh-Sulfatmit } \\
1 / 2 \% \text { Cinchonidinsulfat } \\
\text { verrieben. }{ }^{\circ} \text {. } \\
\text { Je } 5 \text { C. C. mit je } 0,0025 \text { Cin- } \\
\text { chonidinsulfat gebrauchen. }\end{array}$ & $\begin{array}{l}4,05 \\
4,05 \\
4,10 \\
4,05\end{array}$ & $\begin{array}{l}6,10 \\
6,10 \\
6,17 \\
6,15\end{array}$ & & & & \\
\hline Durchschnittl. & 4,06 & 6,13 & 0,75 & 0,87 & $0, \mathbf{3 0}$ & 0,34 \\
\hline 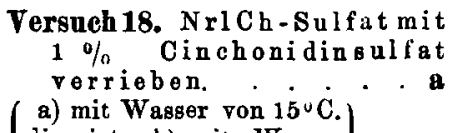 & $\begin{array}{l}5,00 \\
5,00 \\
4,95\end{array}$ & $\begin{array}{l}7,50 \\
7,45 \\
7,50\end{array}$ & & & & \\
\hline $\begin{array}{l}\left\{\begin{array}{l}\text { digerirt, b) mit Wasser } \\
\text { von } 60^{\circ} \mathrm{C} \text {. c) mit Wasser } \\
\text { von } 80^{\circ} \mathrm{C} \text {. (b u. c vor d. } \\
\text { Filtriren aut } 15^{\circ} \mathrm{C} \text {. abgek.) }\end{array}\right\} \\
\text { Je } 5 \text { C. C. mit je } 0,005 \text { Ci }- \\
\text { chonidin sulf.gebrauchen }\end{array}$ & $\left\{\begin{array}{l}4,98 \\
5,02 \\
4,95 \\
5,00\end{array}\right.$ & $\begin{array}{l}7,50 \\
7,50 \\
7,50\end{array}$ & 1,67 & 2,22 & $0, \mathbf{3 3}$ & 0,44 \\
\hline Dschn. & $\begin{array}{l}\mathbf{4 , 9 9} \\
\left\{\begin{array}{l}5,00 \\
5,05 \\
5,00\end{array}\right. \\
\end{array}$ & $\begin{array}{l}\mathbf{7 , 5 0} \\
\mathbf{7 , 5 0} \\
7,53 \\
\mathbf{7 , 5 0} \\
\end{array}$ & 1,68 & 2,24 & $0, \mathbf{3 3}$ & 0,44 \\
\hline Dsehn. & 5,01 & $7, \overline{51}$ & 1,70 & 2,25 & $\mathbf{0 , 3 4}$ & 0,45 \\
\hline
\end{tabular}

III. Versuchsreihe. Nach den Resultaten der Versuche 1-18 erübrigt nur noch die Beweisführung, dass H ess e's Probe für solche Nachweise, wie sie hier geliefert sind, vollkommen im Stiche lässt. Man stelle 4 Verreibungen von chem. reinem Chininsulfat mit Cinchonidinsulfat her, wie bei den Versuchen 15 bis 18 angegeben und bereite daraus Wasserauszüge genau nach Hesses Recept (im Verhältniss von 1:20). Mit diesen Lösungen führe man nun einerseits die Hesse'sche Probe aus, andererseits benütze man je 5 C.C. derselben (auf $15^{\circ} \mathrm{C}$. gestellt) zur genauen Ammoniakprobe (resp. Titrirung). 


\begin{tabular}{|c|c|c|c|c|}
\hline & $\begin{array}{l}\text { Hesse's } \\
\text { Probe' }\end{array}$ & $\mid \begin{array}{c}\text { Verbraucht } \\
\text { C. C. Ak } \\
\text { von } 0,92 \\
\text { zu } 5 \text { C. C. } \\
\text { Chininausz. }\end{array}$ & $\begin{array}{c}\text { Mehrver- } \\
\text { brauch an } \\
\text { C. C. Ak von } \\
0,92\end{array}$ & $\begin{array}{l}\text { Je } 1 \text { Millig. } \\
\text { Cinchoni- } \\
\text { dinsulfat } \\
\text { erforderten } \\
\text { C.C. } A k 0,92\end{array}$ \\
\hline $\begin{array}{l}\text { Versuch 19. Normalchin- } \\
\text { auszug (1 à 20) } \\
\text { (Titrestellung d. Ak-Liquors) }\end{array}$ & $\begin{array}{c}\text { selbstrer- } \\
\text { ständlich keine } \\
\text { Reaction }\end{array}$ & $\begin{array}{l}3,30 \\
3,30\end{array}$ & - & - \\
\hline $\begin{array}{l}\text { Versuch 20. NriCh-Sul- } \\
\text { fat }+1 / 10 \% \text { Cinchoni- } \\
\text { dinfulfat } \\
\text { Je } 5 \text { C.C. mit 0,00025 } \\
\text { Cinchonidingulfat. }\end{array}$ & $\begin{array}{c}\text { nach } 2 \text { Ta- } \\
\text { gen noch klare } \\
\text { Aetherschichte } \\
\text { ohne alle } \\
\text { Krystalle }\end{array}$ & $\left.\begin{array}{l}3,40 \\
3,40\end{array}\right\}$ & 0,10 & 0,40 \\
\hline $\begin{array}{l}\text { Versuch 21. NrlCh-Sulfat } \\
+1 / \% \text { Cinchonidsul. } \\
\text { Jo } 5 \mathrm{C} \text {. C. mit } 0,000625 \\
\text { Cinchonidinsulfat. }\end{array}$ & $\begin{array}{l}\text { nach } 2 \text { Ta- } \\
\text { gen keiner- } \\
\text { lei Krystalle } \\
\text { sichtbar }\end{array}$ & $\left.\begin{array}{l}3,55 \\
3,55\end{array}\right\}$ & 0,25 & 0,40 \\
\hline $\begin{array}{r}\text { Versueh 22. NrlCh-Sulfat } \\
+1 / 2 \% \text { Cinchonidsulf. } \\
\text { Je C. C. mit 0,00125 } \\
\text { Cinchonidinsulfat. }\end{array}$ & $\begin{array}{c}\text { nach } 2 \mathrm{Ta}- \\
\text { gen noch } \\
\text { ohne } \\
\text { Krystalle }\end{array}$ & $\left.\begin{array}{l}3,70 \\
3,70\end{array}\right\}$ & 0,40 & 0,32 \\
\hline $\begin{array}{l}\text { Versuch 23. NrlCh-Sulfat } \\
+1 \% \text { Cinchnid.-Sulf. } \\
\text { Je } 5 \text { C. C.mit } 0,0025 \text { Cin- } \\
\text { chonidinsulfat }\end{array}$ & $\begin{array}{c}\text { nach } 2 \mathrm{Ta}- \\
\text { gen noch } \\
\text { keine } \\
\text { Krystalle }\end{array}$ & $\left.\begin{array}{l}4,10 \\
4,05\end{array}\right\}$ & 0,77 & 0,30 \\
\hline
\end{tabular}

Die Ammoniakprobe giebt also noch einen beträchtlichen Ausschlag (mit quantitativem Ergebniss) wo die Aetherausschüttlung der Lösungen nach Hesse absolut resultatlos bleiben, allein trotz solcher Beweise, wie die Vorstehenden, die in anderer Anordnung auch bereits aus meiner früheren Abhandlung ersichtlich sind, besteht Hesse dreist auf der Behauptung, seine Probe könne noch Cinchonidin im Chininsulfat diagnosticiren, wo die Ammoniakprobe gänzlich fehlschlage. Da das directe Experiment Jedermann von dem Gegentheil überzeugen muss, so versucht Hesse nun die neue Erklärung, das Cinchonidin sei unter Umständen im Chininsulfat "latent gebunden" und könne deshalb der Ammoniakprobe entgehen,

1) Der verwendete Aether war ein wiederholt rectificirtes Präparat. Die Ausschüttlung damit wurde in langen engen Reagensgläsern mit Theilstrichen für $5+1$ C.C. vorgenommen; eine andere Bezeichnung kann ich für diese Röhren nicht finden, obschon es Hesse für ,absurd “ erklärt, dass ich darin keine eigen thümlichen "Chininometer" erkennen kamn. 
es ist aber doch einleuchtend, dass, wenn an der Geschichte von dem „latenten Cinchonidin“ (vielleicht entpuppt sich daraus noch ein neues Alkaloïd ?) wirklich etwas Wahres wäre, dieser Einwand mindestens ebenso gegen die Hesse'sche Probe sprechen müsste, welche sich ja ebenfalls auf Chininsulfatwasserauszüge stützt! Krystallisirt man grössere Mengen von Cinchonidinsulfat mit Chininsulfat zusammen, so resultiren allerdings Doppelsalze bei der Alkaloïde (das Cinchonidinsulfat krystallisirt gewissermassen in das Chininsulfat hinein) und ist es auch nicht möglich aus solchen Salzen sämmtliches Cinchonidin durch einmaliges Extrahiren mit der 10-20fachen Menge Wasser auszuziehen; allein werden solche Verbindungen in Verhältniss der Ammoniakprobe (1 à 10) mit Wasser verrieben; so bleiben die Auszüge auf Zusatz von Ammoniak (5C.C. pro 5 C. C.Ak 0,92, oder 7 C.C. 0,96) stets ganz milchig trübe, und geben die Verunreinigung also sehr intensiv zu erkennen. In diesen Fällen zeigt auch schon die alte Liebigsche Probe (wobei die ganze Substanz mit Aether und Ammoniak ausgeschüttelt wird) das Cinchonidin deutlich an und ist hierbei jedenfalls sicherer als die Hesse'sche Veränderung. Warum überhaupt aus Chininsulfat mit $9-13 \%$ Cinchonidinsulfat von dem Letzteren durch Zerreiben der Substanz mit der 10 fachen Menge Wasser Nichts in Lösung gehen soll, ist ganz unverständlich, da es $7 \mathrm{mal}$ löslicher ist als schwefelsaures Chinin; - und dass, wenn einmal auch nur Spuren davon in den Auszug gelangt sind, solche durch meine Probe aufs Schärfste nachzuweisen sind, geht doch wahrlich aus den vorstehenden Versuchen unzweifelhaft hervor! - Hessc will seine Erfindung von dem Chininsulfat mit $13 \%$ "latent" gebundenem Cinchonidinsulfat durch eine neue "optische“ Probe erzielt haben. Da diese Probe aber ebenfalls noch "latent", d. h. bis jetzt unbekannt ist, so ist eine solche Beweisführung zunächst werthlos; - möglicherweise liegt eine optische Täuschung zu Grunde.

Wir verdanken Oudemanns bereits ganz fein ausgearbeitete optische Bestimmungsmethoden für Chinaalkaloüdgemische und dürfte an denselben Nichts zu, , verbessern"6 sein. Derartige analytische Wege sind' aber nur für sehr geübte Hände und können kaum zu Handelsproben empfohlen werden. Es ist unstreitig, dass unter einzelnen Chininsulfaten diejenigen am besten sind, welche, unter ganz gleichen Verhältnissen beobachtet, am meisten Arch. d. Pharm. XVII, Bds. 6. Hft. 
links drehen, gleichwohl dürfte es schwer halten, Verunreinigung im schwefelsauren Chinin mit Cinchonin, Chinidin und namentlich mit dem links drehenden Cinchonidin scharf zu bestimmen, wenn es sich nur um $1 \frac{1}{2}, 1 \%$ dieser Alkaloïdsulfate oder darunter handelt.

Officinelle Proben müssen so construirt sein, dass sie von allen Droguisten, Apothekern und Medicinalbeamten gleichmässig zuverlässig und scharf vorgenommen werden und bezüglich des Resultates keinerlei Zweifel oder Missverständnisse a fkommen lassen können!

Ueber die verschiedenen Möglichkeiten, welche bei dem Hesseschen Verfahren zu Irrthümern und falscher Beurtheilung führen können, habe ich schon in meiner ersten Abhandlung eingehend gesprochen. Hesse vermeint, diese Bedenken mit seiner Erwiderung beseitigt zu haben, muss aber selbst zugeben, dass z. B. das jede Beobachtung illusorisch machende Gelatiniren der Aetherschichten bei manchen Aethersorten des Handels und der Apotheken ${ }^{1}$ vorkommen $\mathrm{kann}$, und in der That war es mir auch in mehreren Fällen möglich, falsche Urtheile über Chininsulfate, welche man nach Hesse als nicht probehaltig befunden haben wollte, direct auf $\mathrm{Täuschungen} \mathrm{dieser} \mathrm{Art} \mathrm{zurückzuführen.} \mathrm{Wenn} \mathrm{in}$ Hesse's Probe auch nur in irgend einer Hinsicht eine nachweisbare Verbesserung gegenüber der Ammoniakprobe läge, würde ich nicht anstehen, seine Methode selbst zu empfehlen; da aber bei derselben

1) Irrthümer und Täuschungen keineswegs ausgeschlossen sind,

2) eine sofort erkennbare zweifellose Endreaction nicht besteht, und

3) eine auch nur annähernd modifirbare quantitative Schätzung unmöglich ist,

1) Als Ursache des Gelatinirens der Chininätherschichten sieht Hesse den etwaigen Gehalt des officinellen Aethers an Sohwefligersäure an. Es ist möglich, dass dies in einzelnen Fällen der Grund sein $k a n n$, aber es ist offenbar nicht die ausschliessliche Ursache dieses gewichtigen Missstandes. Ich habe wiederholt gefunden, dass derselbe auch bei Aether, welcher allen Anforderungen der Pharmacopöe (also auch bezüglich der Freiheit vom $\mathrm{SO}^{2}$ oder $\mathrm{SO}^{3}$ ) entspricht, und zwar bei sehr reinem Chininsulfat eintreten kann. Wenn dies Hesse wirklich noch nicht beobachten konnte, trotz seiner 500 Prüfungen, so war daran wohl nur der Zu fall schuld, dass er stets gleichmässigen und ganz reinen Aether zur Hand hatte. 
bei der officinellen Ammoniakprobe dagegen

1) eine falsche, ungerechte Beurtheilung nicht vorkommen kann,

2) die Endreaction alsbald bei Anstellung der Probe unanfechtbar eintritt, und

3) in der Methode selbst (wie Oben gezeigt) die Möglichkeit liegt, Anwesenheit von Nebenalkaloüden sogar unterhalb $1 \%$ noch anähernd quantitativ nachzuweisen und in dieser Weise noch zwischen verschiedenen probehaltigen Präparaten scharfe Vergleiche anzustellen,

so ist wahrlich nicht einzusehen, warum diese eingebürgerte Methode nicht mehr genügen soll! Hesse's persönlicher Drang und Wunsch seinerseits, à tout prix eine eigene neue Probe in die Welt setzen zu wollen (womit nebenbei sonstige Reclame getrieben werden soll), dürfte doch für die rein objectiven maassgebenden Beurtheiler nicht bestimmend wirken.

Wie ich schon früher ausführlich erörterte, muss für eine officinelle Chininsulfatprobe auf Nebenalkaloïde eine bestimmte Grenze mit klarem Maassstab gestellt sein, welche vernünftigerweise gefordert werden darf und darum auch gefordert werden muss. Für den practischen Arzt und die Heilungsbedürftigen ist es durchaus irrelevant, ob und wenn das in den Apotheken verabreichte Chinin einen zulässigen Maximalgehalt von $1 \%$ an einem oder dem anderen der jedenfalls sehr ähnlich wirkenden Nebenalkaloïde enthält, und einen solchen Gehalt zeigt die Ammoniakprobe deutlich (an ihrer jetzigen Grenze), Hesse's Methode aber theils gar nicht, theils erst nach längerer Zeit an. Die Bemessung des inneren Werthes und zugleich der W irksamkeit des Chinins hängt von einem ganz anderen Umstande ab, dem früher (d. Arch. Bd. XIII. pag. $201-205$ ) geschilderten wechselnden Gehalte an Krystallwasser und mechanischer Feuchtigkeit, der öfters nach vielen Procenten schwankt. Ich habe deshalb vorgeschlagen, entweder das constante Chininsulfat mit 2 Aequival. Krystallwasser officinell zu machen, was auch Professor van der Burg in Leyden gleichzeitig (ganz unabhängig von mir) empfohlen hat, oder doch wenigstens für das Cetzt gebräuchliche schwefelsaure Chinin nur einen Maximalgehalt von $14,6 \%$ bei $115^{\circ} \mathrm{C}$. verjagbaren Wassers zuzulassen. Hesse zollt dieser Ansicht keinen Beifall und tritt für hochgewässertes Chinin in die Schranken. Er nennt ein Chininsulfat 
mit $11 \%$ Krystallwasser, das die Ammoniakprobe hält, "sogenanntes" gutes Chinin und ich glaube, Aerzte und Pationten würden ihm gewiss noch weitergehend beistimmen und zu berechnen verstehen, dass ein derartiges Präparat in der 'That auch weit mebr werth ist und ergiebiger wirkt, als ein solches mit 16-18 Procent Wasser. Wünscht der Arzt Wasserbehandlung neben Chinin, so kann er dem Kranken billigere Wege zeigen; - die Heilkraft und der Geldwerth eines Chininsulfates hängen in erster Linie von seinem Procentgehalte an reinem Alkaloüd (Chinin) ab!

Ich resumire daher meine früheren und obigen Auseinandersetzungen dahin:

I. Man verlange bei der Werthbemessung eines käuflichen Chinin. sulfuric. vor Allem, dass es mindestens $74 \frac{1}{4}$ Procent reines wasserfreies Chinin (bei $115^{\circ} \mathrm{C}$. getrocknet) enthalte. Entweder lasse man diesen Gehalt direct bestimmen, oder nehme die einfachere, regelrechte Austrocknung bis $115^{\circ} \mathrm{C}$. (wie a. a. 0 . pag. 205 beschriebon) vor und gestatte hierbei nur einen Maximalgewichtsverlust bis 14,6 Procent.

II. Die Prüfung auf Nebenalkaloïde ist (präcise gefasst) auszufiihren, wie folgt: Man verbringe $2 \mathrm{~g}$. des fraglichen Präparates, fein zerrieben, in eine kleine Flasche, setze 20 g. lauwarmes destillirtes Wasser zu, verschliesse das Gefäss mit einem Stopfen und schüttele öfters kräftig un. Nach $1 / 2$ Stunde stelle man die Flasche in ein Becken mit kaltem Brunnenwasser und filtrire den Inhalt, wenn er die Normaltemperatur von $15^{\circ} \mathrm{C}$. auf einem eingesteckten Thermometer angiebt, nach wiederholtem Schütteln durch ein trockenes Filter. Von dem Filtrate giesse man genau 5 C.C. in einen in zehntel C. C. getheilten 10 C.C. Cylinder ${ }^{2}$, füge 3 C. C. starken Ammoniak -

1) $1 / 2^{\circ}$ C. über oder unter der Normaltemperatur von $15^{\circ} \mathrm{C}$. hat keinen deutlichen Einfluss, daher das Einstellen auf die Normaltemperatur nicht so ängstlich gehandhabt zu werden braucht. Der Verbrauch an Chinin für die Probe ist ganz geringfügig, da man das auf dem Filter verbleibende Präparat wieder luftrocken machen und dispensiren kann.

2) Solche Cylinder sind in allen grösseren Handlungen chem. und pharmaceutiseher Apparate vorräthig und können in pharmac. Laboratorien für die verschiedensten Zwecke nützlich sein. 
liquor ${ }^{1}$ von 0,92 sp. G. zu, verschliesse das Gefäss mit dem Finger nnd schwenke einmal um. Zu dem meist noch trüben Gemischesetze man nun tropfenweise weiteren Ammoniakliquor unter jedesmaligem einmaligen Um. schwenken, bis vollkommene Klarheit eintritt (d. h, die letzte Spur von Färbung verschwindet, was auf den Tropfen genau zu treffen ist). Es darf hierzu im Ganzen höchstens 5 C.C. des Ammoniakliquors ron $0,92 \mathrm{sp}$. Gew. nöthig sein.

Mit diesen beiden reellen und concisen Ansprüchen an ein officinelles Chininsulfat ist rom practisch pharmacologischen Gesichtspunkte, der hierbei allein maassgebend sein kann, ein vollk ommenes Ge nüge geleistet. ${ }^{2}$

Im Novemberheft dieses Archivs (pag. 369) findet sich noch die Notiz:

„O. Hesse hält seine frühere Angabe, dass chem. reines Chininsulfat mit 8 Moleculen, $=16,17 \%$ Wasser krystallisirt, aufrecht. Unverwittertes Chininsulfat mit weniger Wasser deutet sofort anf eine Beimischung von Cinchonidinsulfat, welches mit nur $6 \mathrm{H}^{8} \mathrm{O}$ krystallisirt."

Man vergleiche hiergegen meine Ausfübrungen pag. 202-204 im Märzhefte dieses Archivs. Ich habe dort ausdrücklich eingeränmt, dass Chininsulfate mit mehr als $7 \mathrm{Mol} . \mathrm{H}^{2} \mathrm{O}$ existiren können, zugleich aber betont, dass diese Frage wesentlich eine practische und nicht als Formelfrage aufzufassen sei. Es ist nicht mög lich, das sehr voluminöse Chininsulfat des Handels practis ch so zu trocknen, dass es ohne bereits beginnende Verwitterung ganz von anhängender Feuchtigkeit frei ist. Mit Recht kann und muss jedoch verlangt werden, dass der Handelswaare ke ine mechanische Feuchtigkeit anhänge! Die Erfahrung von einem halben Jahrhundert hat aber gelehrt, dass gutes, von mechanischer Feuchtigkeit freies Chinin des Handels nicht mehr als $14-14,6 \%$ bei $115^{\circ} \mathrm{C}$. verliert, was der For-

1) Hat man nur den bisher officinellen schwächeren Ammoniakliquor von 0,96 zur Verfügung, so kann derselbe ebenfalls dienen. Man hat dann auf 5 C.C. Chininauszug zuerst 5 C. C. des schwachen Liquors zuzusetzen und einen Maxima lverbrauch ron rot. 7 C.C. desselben als lässig anzusehen.

2) Selbstverständlich wird eine vorgängige allgem eine Prifung auf Fremdkörper (beträgerische Verfälschung mit $\mathrm{MgO}$, Salicylsäure, sohwefelsaures Ammoniak etc.) vorausgesetzt. 
mel mit $7 \mathrm{H}^{2} \mathrm{O}$ entspricht. Bei höher gewässerter Handelswa are kann meist mit Leichtigkeit (durch rasches Pressen zwischen Filtrirpapier) constatirt werden, dass dieses Plus von Wasser nicht von Krystallwasser, sondern von anhängendem Wasser herrührt. Ich beharre daher auf $\mathrm{m}$. Vorschlage, als Maximum des zulässigen Trockenverlustes $14,6 \%$ festzuhalten. Die Gestattung von $16 \%$ würde nur bewirken, dass die 2te Hand des Handels es unter Umständen der Mühe werth halten kann, gut trockenes Handelschinin (das in den seltensten Fällen üb er 141/2\% Wasser abgiebt) durch vorsichtiges Besprengen auf $16 \%$ und mehr zu wässern, -- ein Vorkommniss, das ich in der That unlängst constatiren konnte. Hesse's Insistiren auf den 16,17\% Wasser entspricht also sicher nicht dem Zwecke, diese pharmacologische Frage real zu lösen.

Wenn Hesse ferner geltend machen will, dass ein Chininsulfat mit weniger als $16 \% \mathrm{H}^{2} \mathrm{O}$ sofort auf eine mögliche „Beimischung" von Cinchonidinsulfat hinweise, so ist dies durchaus irrelevant. Durch vorstehende Mittheilungen glaube ich doch endlich zur Genüge bewiesen zu haben, dass ein schwefelsaures Chinin, welches die Ammoniakprobe (in den angegebenen Verhältnissen) hält, höchstens $1 \%$ Cinchonidinsulfat enthalten $\mathrm{kann}$; - und dass dieses $1 \%$ in einem fraglichen Chininsulfat einen Mindergehalt an Krystallwasser von $11 / 2,2 \%$ und mehr veranlassen kann und soll, wird Niemand im Ernste glauben.

Frankfurt a. M., den 1. December 1880.

\section{B. Monatsberich t.}

Ist ein Zusatz von Salicylsăure zu Wein und Bier polizeilich $\mathrm{zu}$ verbieten und strafrechtlich zu ahnden?

A potheker A. Huber hatte sich dieses Thema zu einem Vertrage, gehalten in der Jahresversammlung des schweizerischen Apothekervereins, gewälilt und leitete denselben mit einem Auspruche von Justus Liebig von Jahre 1853 ein: „Die Natur erzeugt seinen Wein; es ist immer der Mensch, der ihn fabricirt, der durch die kïnstlichen Mittel der sogen. Veredlung die Naturkräfte nach seinen Zwecken lenkt und wirken lässt. Ich bin überzeugt, dass in einem Menschenalter in schlechten Jahrgängen die Verbesserung des Mostes durch Zucker längs des ganzen Rheines ganz allge- 